\title{
New-onset neonatal pulmonary hypertension associated with a rhinovirus infection
}

\author{
Nishit Patel MD, Tiong G The MD
}

N Patel, TG The. New-onset neonatal pulmonary hypertension associated with a rhinovirus infection. Can Respir J 2012;19(1): 33-34.

A 3.5-week-old male neonate who developed an upper and lower respiratory tract rhinovirus infection that was temporally associated with the development of severe pulmonary hypertension is described. Rhinovirus has not previously been associated with pulmonary hypertension. This child developed severe pulmonary hypertension with right ventricular failure, requiring mechanical ventilation, nitric oxide inhalation and, eventually, extracorporeal membrane oxygenation.

\section{Une hypertension pulmonaire néonatale de novo associée à une infection par le rhinovirus}

Les auteurs décrivent le cas d'un nouveau-né de 3,5 semaines de sexe masculin qui a contracté une infection des voies respiratoires supérieures et inférieures par le rhinovirus, laquelle était liée dans le temps à l'apparition d'une grave hypertension pulmonaire. Le rhinovirus n'a pas été associé à l'hypertension pulmonaire auparavant. Ce nourrisson a présenté une hypertension pulmonaire grave accompagnée d'une insuffisance ventriculaire droite exigeant une ventilation mécanique, l'inhalation d'oxyde nitrique, puis une oxygénation extracorporelle.

Key Words: ECMO; Neonate; Pulmonary hypertension; Rhinovirus; Right ventricular failure

\begin{tabular}{|c|c|}
\hline 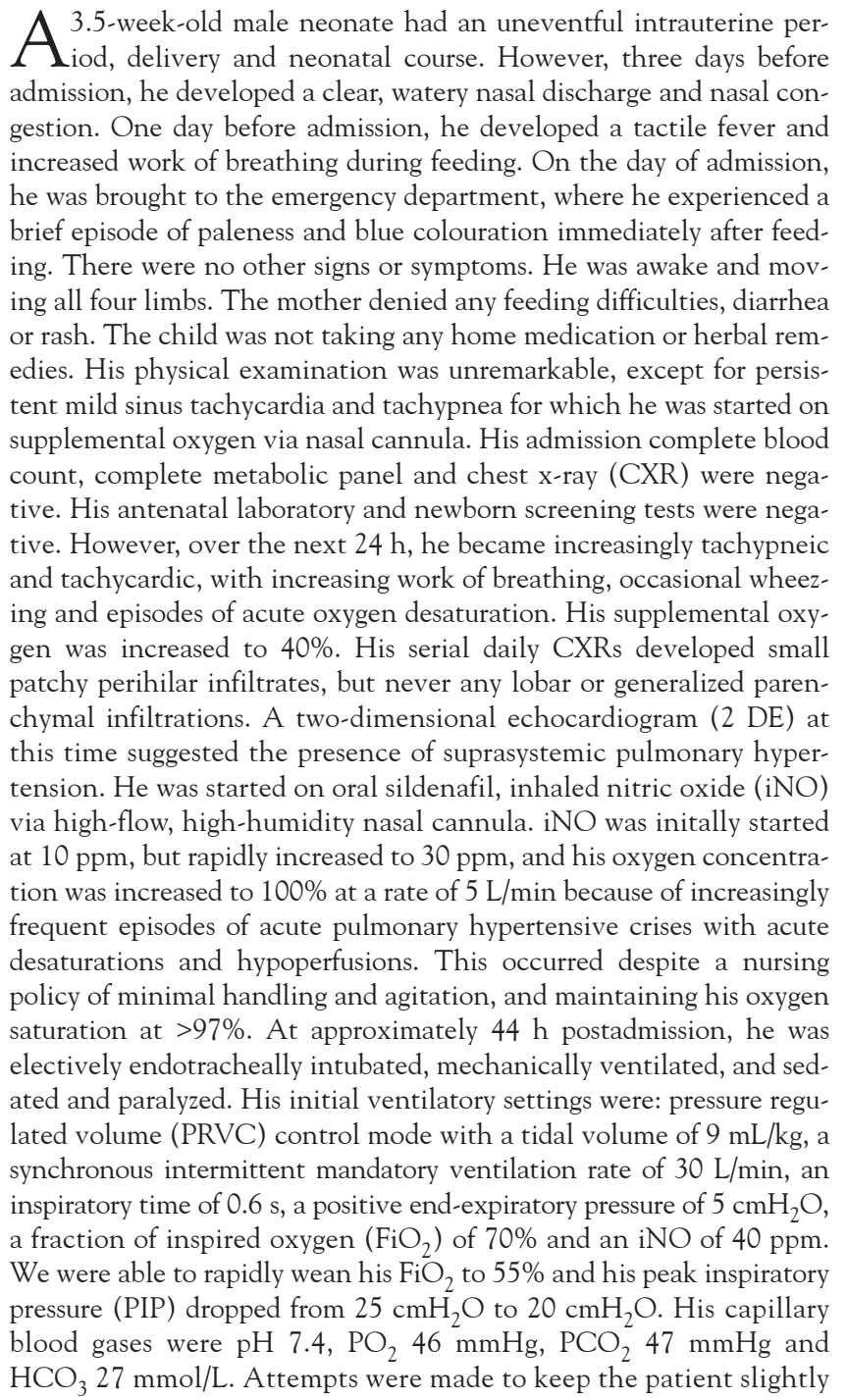 & 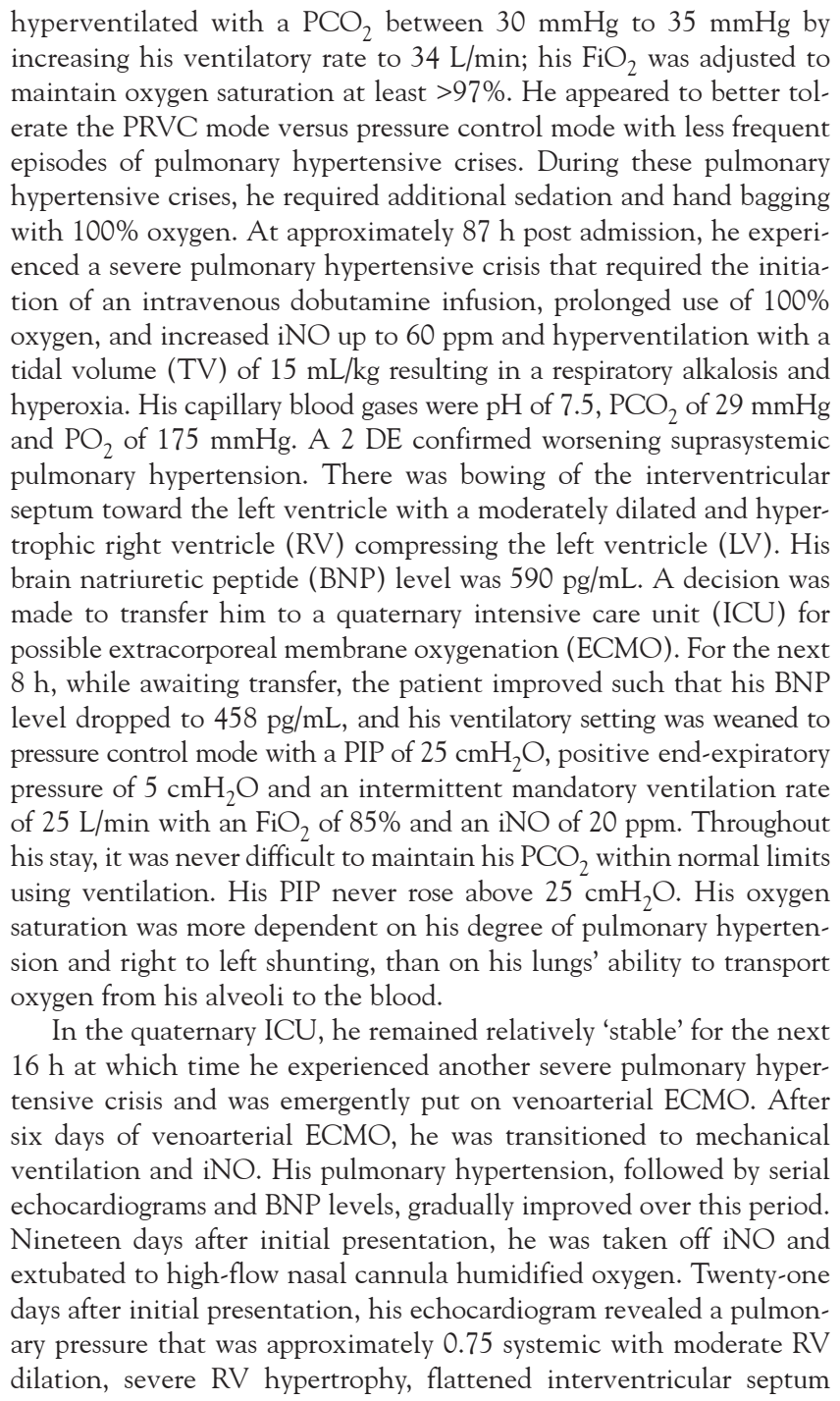 \\
\hline
\end{tabular}

${ }^{1}$ St Peter's University Hospital, 254 Easton Avenue, New Brunswick, New Jersey, USA

Correspondence and reprints: Dr Tiong G The, Division of Pediatric Critical Care, St Peter's University Hospital, 254 Easton Avenue,

New Brunswick, New Jersey 08901, USA. Telephone 732-745-8600 ext 8783, fax 732-745-0857, e-mail tthe@saintpetersuh.com,

nishitpatel@saintpetersuh.com 
and persistence of the mild tricuspid regurgitation and patent foramen ovale with bidirectional shunting. There was a new right atrial pericardial effusion. Qualitatively, the RV diastolic and systolic function remained markedly decreased; the LV systolic function was normal. Forty-five days after initial presentation, computed tomography angiography and cardiac catheterization revealed increased pulmonary resistance, but subsystemic pressures that were not responsive to NO or oxygen, normal pulmonary veins and possible ground-glass atelectasis. The overall picture was believed to suggest mixed lung disease and pulmonary hypertension. Seventy-three days after the initial presentation, an echocardiogram showed further improvement in the patient's pulmonary hypertension, with only mild to moderately diminished RV systolic shortening, mild to moderate dilation and hypertrophy of RV, and flattened ventricular septum in relation to LV. His BNP level had fallen to $161 \mathrm{pg} / \mathrm{mL}$. Seventy-eight days after initial presentation, he was discharged home on oral sildenafil and $1 \mathrm{~L} / \mathrm{min}$ oxygen via nasal cannula.

The following day, the patient was readmitted for overnight observation because of an accidental overdose of sildenafil. He was feeding well, and taking full oral bolus feeding. His physical examination was within normal limits, without any signs or symptoms of an upper respiratory infection.

The following laboratory findings were significant: his respiratory virus polymerase chain reaction (PCR) panel was positive for rhinovirus; it was negative for respiratory syncytial virus (RSV), influenza A and $\mathrm{B}$, adeno, parainfluenza and meta-pneumo viruses (a study with a $99 \%$ sensitivity rate); all bacterial cultures were negative; his milk scan and barium swallow showed mild clinically insignificant gastroesophageal reflux up to the mid esophagus without any aspiration; testing for surfactant deficiency was negative; and brain magnetic resonance imaging results were within normal limits.

\section{DISCUSSION}

We believe that the rhinovirus infection was the cause of this child's new-onset pulmonary hypertension. If this child had persistent pulmonary hypertension of the newborn, he would have presented much earlier in life and most likely with a more difficult neonatal course. Instead, he was a healthy newborn who had gained $650 \mathrm{~g}$ since birth. He presented with a three-day history of upper respiratory tract infection symptoms and a medically witnessed transient episode of cyanosis, which in retrospect, could have been due to acute right to left shunting through his still patent foramen ovale or acute right heart decompensation due to suprasystemic pulmonary hypertension. His essentially normal initial physical examination, CXRs and laboratory findings, including negative bacterial cultures, are consistent with this hypothesis. His persistent tachypnea, tachycardia and elevated BNP level were clues to his worsening right heart failure due to severe pulmonary hypertension. This was confirmed on his 2DEs. His worsening clinical course and subsequent improvement also support the hypothesis that his condition was related to an acute illness. His acutely worsening pulmonary hypertension in the first week of presentation coincided with the rapid replication phase of a rhinovirus infection. The amelioration of his suprasystemic pulmonary hypertension to subsystemic levels at three weeks after initial presentation was also consistent with the natural history of rhinovirus infection. Rhinovirus becomes minimally detectable in respiratory tissues after three weeks of infection, although it still can be detectable up to five weeks after infection when the antibodies levels to rhinovirus rise to a peak (1). The only positive finding found after extensive investigation in our patient was the presence of rhinovirus in the PCR respiratory panel. We did not repeat his respiratory panel on his second admission because he did not exhibit any symptoms of a respiratory tract infection and because by 79 days after his initial infection, his rhinoviral PCR panel should have become negative.
Rhinovirus has been associated with up to $35 \%$ of 'common colds'. However, to the best of our knowledge, rhinovirus has not been associated with pulmonary hypertension. Some serotypes of rhinovirus have been shown to infect the lower respiratory tract (1). Fitzgerald et al (2) reported evidence of pulmonary hypertension in four of six mechanically ventilated infants with acute RSV bronchiolitis. Castelli et al (3) reported a nine-month-old infant who died on the fifth postoperative day after surgical correction for aortic coarctation, with clinical and autopsy evidence of acute bronchiolitis and severe pulmonary hypertension and immunohistochemistry and electron microscopy evidence of RSV in the pulmonary tissue (3). There was good repair at the site of aortic coarctation. The development of small patchy perihilar infiltrates and wheezing in our patient was consistent with the development of acute bronchiolitis. Other viruses have been associated with the development of pulmonary hypertension, notably congenital echovirus 11 (4). Severe pertussis has also been associated with neonatal pulmonary hypertension (5), but our patient did not demonstrate any of the characteristic whooping cough or lymphocytosis. Severe bronchopneumonia has also been associated with pulmonary hypertension (6), but our patient did not show any evidence of this on CXRs. Seki et al (7) reported the first association of severe gastroesophageal reflux and aspiration pneumonia with the development of pulmonary hypertension in a two-month-old boy with Down's syndrome (7). Our patient did not have clinically significant gastroesophageal reflux. In fact, on resuming full oral bolus feeding, his pulmonary hypertension had improved.

\section{SUMMARY}

We believe that our patient had developed an acute upper and lower respiratory tract rhinovirus infection that was temporally associated with the development of severe pulmonary hypertension. The time course of the amelioration of his pulmonary hypertension was also consistent with a prolonged rhinovirus infection. However, given the wide prevalence of rhinoviral infections, we should be cautious in ascribing a direct cause and effect relationship. We should, however, be aware of its possible association, and look for additional cases to be reported.

The institutional review board of St Peter's University Hospital approved this project (CPHSR study \# 10:47) and provided exemption of informed consent.

DISCLOSURES: The authors did not receive any funding from any agency for this work and do not have any conflicts of interest to declare.

\section{REFERENCES}

1. Brownlee JW, Turner RB. New developments in the epidemiology and clinical spectrum of rhinovirus infections. Curr Opin Pediatr 2008;20:67-71.

2. Fitzgerald D, Davis GM, Rohlicek C, Gottesman R.

Quantifying pulmonary hypertension in ventilated infants with bronchiolitis: A pilot study. J Paediatr Child Health 2001;37:64-6.

3. Castelli JB, Maeda WT, Aiello VD. Respiratory syncytial virus as a trigger of a pulmonary hypertensive crisis after operative correction of aortic coarctation. Cardiol Young [Case Reports] 2007;17:223-5.

4. Willems A, Benne CA, Timmer A, Bergman KA. Fatal illness associated with pulmonary hypertension in a neonate caused by intrauterine echovirus 11 infection. Am J Perinatol 2006;23:59-61.

5. Sreenan CD, Osiovich $\mathrm{H}$. Neonatal pertussis requiring extracorporeal membrane oxygenation. Pediatr Surg Int 2001;17:201-3.

6. Uner A, Dogan M, Demirtas M, et al. Comparison of nifedipine and captopril in children with pulmonary hypertension due to bronchopneumonia. J Trop Pediatr 2008;54:294-9.

7. Seki M, Kato T, Masutani S, Matsunaga T, Senzaki H. Pulmonary arterial hypertension associated with gastroesophageal reflux in a 2-month-old boy with Down syndrome. Circ J 2009;73:2352-4. 


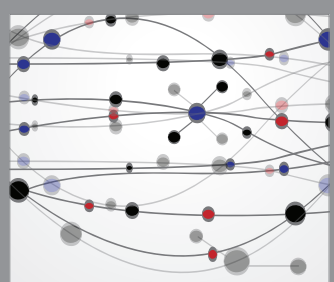

The Scientific World Journal
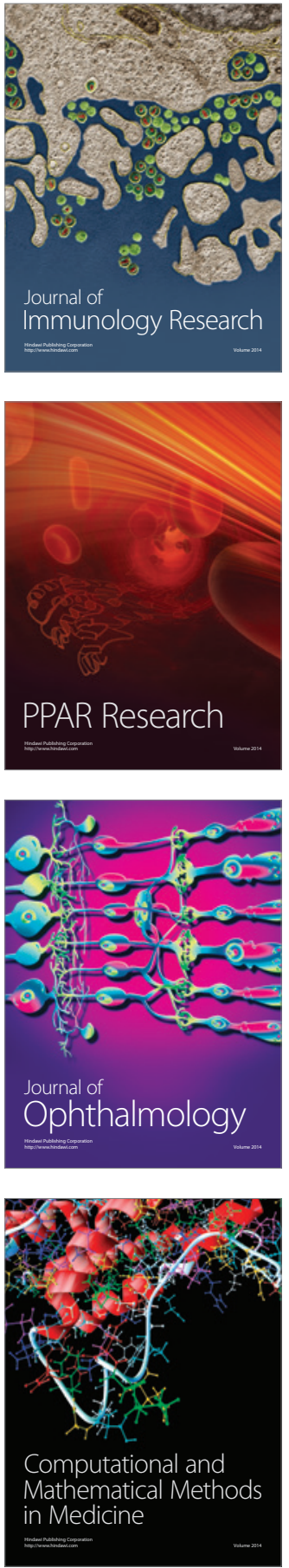

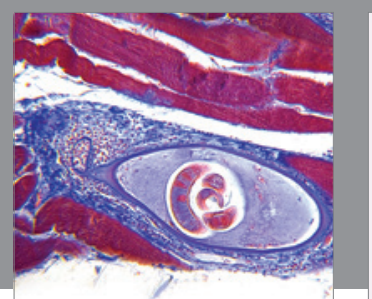

Gastroenterology Research and Practice

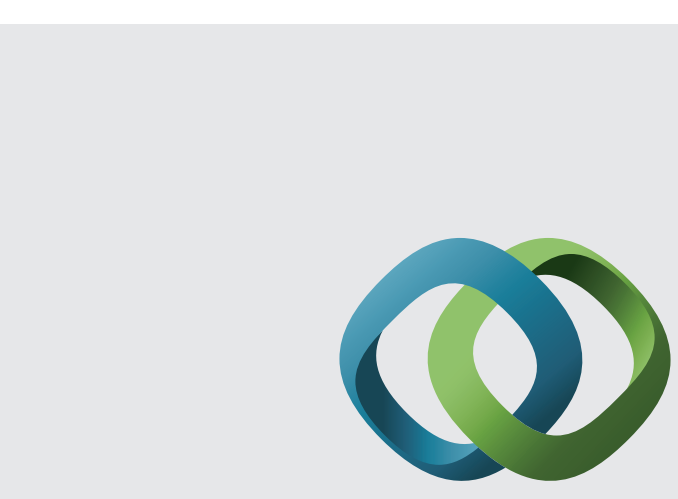

\section{Hindawi}

Submit your manuscripts at

http://www.hindawi.com
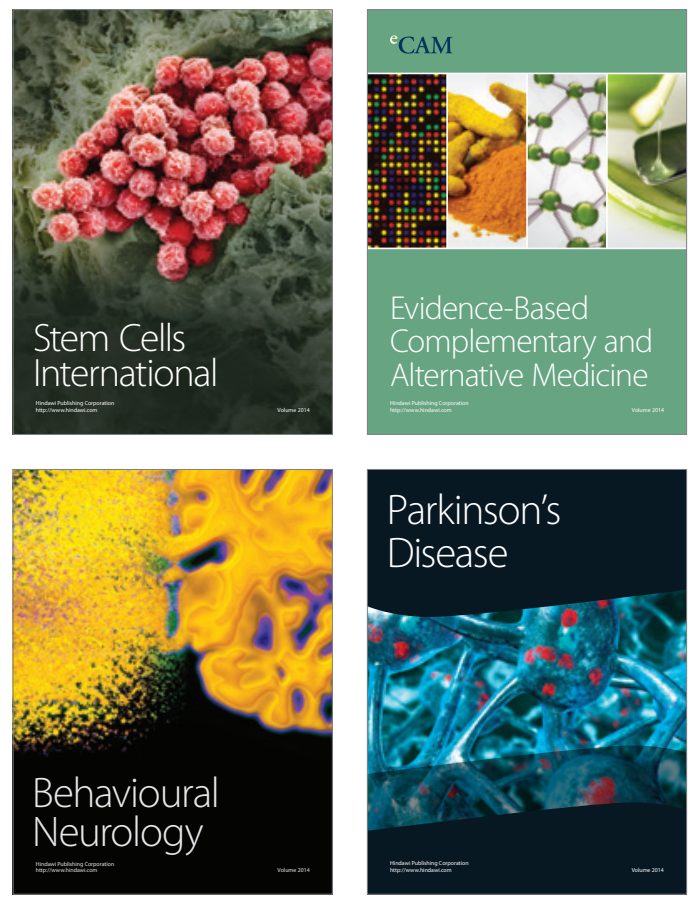
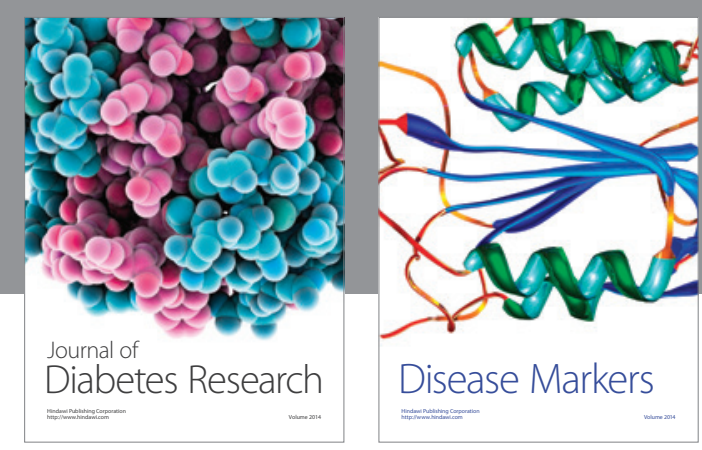

Disease Markers
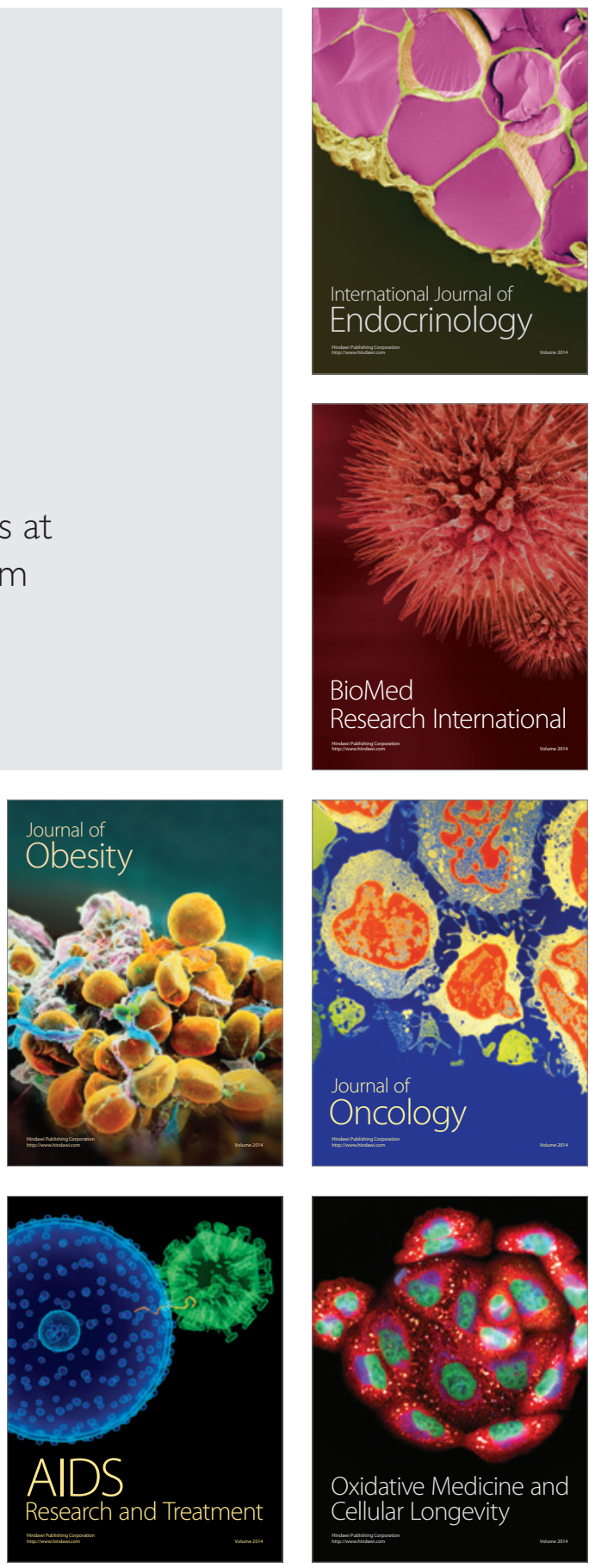